\title{
Genome-wide identification of chitinase genes in Thalassiosira pseudonana and analysis of their expression under abiotic stresses
}

Haomiao Cheng ${ }^{1,2,3}$, Zhanru Shao ${ }^{1,2^{*}}$, Chang Lu ${ }^{1,2,3}$ and Delin Duan ${ }^{1,2,4^{*}}$ (D)

\begin{abstract}
Background: The nitrogen-containing polysaccharide chitin is the second most abundant biopolymer on earth and is found in the cell walls of diatoms, where it serves as a scaffold for biosilica deposition. Diatom chitin is an important source of carbon and nitrogen in the marine environment, but surprisingly little is known about basic chitinase metabolism in diatoms.

Results: Here, we identify and fully characterize 24 chitinase genes from the model centric diatom Thalassiosira pseudonana. We demonstrate that their expression is broadly upregulated under abiotic stresses, despite the fact that chitinase activity itself remains unchanged, and we discuss several explanations for this result. We also examine the potential transcriptional complexity of the intron-rich T. pseudonana chitinase genes and provide evidence for two separate tandem duplication events during their evolution.

Conclusions: Given the many applications of chitin and chitin derivatives in suture production, wound healing, drug delivery, and other processes, new insight into diatom chitin metabolism has both theoretical and practical value.
\end{abstract}

Keywords: Abiotic stress, Chitinase, Enzymatic activity, Gene expression, Gene family, Thalassiosira pseudonana

\section{Background}

Chitin, $\beta$-1,4-linked $N$-acetyl-D-glucosamine, is the second most abundant natural polymer on earth after cellulose. Chitin is found in insect and crustacean exoskeletons, mollusc endoskeletons, and the cell walls of fungi and diatoms [1]. Chitin and its derivative, chitosan, are used in suture production, wound healing, drug delivery, vehicles, scaffolds, and other applications $[2,3]$. Chitin has three main crystal structures: $\alpha-, \beta$, and $\gamma_{-}$ chitin [4]. Among these, $\beta$-chitin displays a parallel

\footnotetext{
* Correspondence: zrshao@qdio.ac.cn; dlduan@qdio.ac.cn

'Key Laboratory of Experimental Marine Biology, Center for Ocean

Mega-Science, Institute of Oceanology, Chinese Academy of Sciences, Qingdao 266071, P. R. China

Full list of author information is available at the end of the article
}

polysaccharide structure that confers greater reactivity, swelling, and solubility compared with the other two chitin types $[5,6]$. $\beta$-chitin is only found in certain marine organisms, including molluscs $[6,7]$, tubeworms [8], and diatoms [9].

Diatoms are dominant primary producers that account for $40 \%$ of primary production in the oceans and perform $20 \%$ of annual global carbon fixation [10]. Chitin is a fundamental structure within the cell walls of diatoms and serves as a supporting scaffold for biosilica deposition [11]. Flexible chitin spines have been proposed to control cell buoyancy or sinking [12], and chitin can form a network around diatom cells that promotes the adhesion of bacteria such as Vibrio parahaemolyticus

C C The Author(s). 2021 Open Access This article is licensed under a Creative Commons Attribution 4.0 International License, which permits use, sharing, adaptation, distribution and reproduction in any medium or format, as long as you give appropriate credit to the original author(s) and the source, provide a link to the Creative Commons licence, and indicate if changes were made. The images or other third party material in this article are included in the article's Creative Commons licence, unless indicated otherwise in a credit line to the material. If material is not included in the article's Creative Commons licence and your intended use is not permitted by statutory regulation or exceeds the permitted use, you will need to obtain permission directly from the copyright holder. To view a copy of this licence, visit http://creativecommons.org/licenses/by/4.0/ The Creative Commons Public Domain Dedication waiver (http://creativecommons.org/publicdomain/zero/1.0/) applies to the data made available in this article, unless otherwise stated in a credit line to the data. 
[13]. As a nitrogenous polysaccharide, chitin is an important source of carbon and nitrogen in the marine environment. It has been suggested that these two elements would quickly disappear from the ocean if chitin stopped circulating $[14,15]$. To date, chitin metabolism has been investigated in two diatom genera, Thalassiosira and Cyclotella, due to the completion of their genome sequencing [16-18].

As known $\beta$-chitin producers, centric diatoms are presumed to contain special enzymes that catalyze chitin biosynthesis and degradation. Chitinases (EC 3.2.1.14) are one of the largest groups of hydrolases and decompose chitin into $N$-acetylglucosamines. Chitinase and its derivatives have been broadly applied in agriculture, pathogenicity, allergenicity, and health [19-21]. Chitinases have been found in diverse organisms from different kingdoms of life, ranging from bacteria and higher plants to animals [22]. They are classified into glycosyl hydrolase families 18 and 19 (GH18 \& GH19) based on the sequence homology of their catalytic domains [23]. GH18 chitinases are widely distributed among organisms such as viruses, bacteria, fungi, yeast, plants, and animals, whereas GH19 chitinases are found almost exclusively in plants [24]. The two chitinase families possess distinct sequence features and different three dimensional (3D-structures, suggesting that they have descended from a different ancestor [25]. Liu et al. (2018) described the sequence homology, domain architectures, and gene expression patterns of chitinases in the oriental fruit fly and proposed their putative physiological functions [26]. Chen et al. (2018) characterized the chitinase gene family in Brassica rapa and examined its role in clubroot resistance [27]. Bartholomew et al. (2019) identified cucumber chitinase genes and characterized their evolution and expression responses to Fusarium oxysporum [28]. Cao \& Tan (2019) studied the tomato chitinase gene family and its response to Sclerotinia sclerotiorum and abiotic stresses including low temperature, high temperature, drought, and salt [29]. Previous work has focused on chitinase functions in nutrition, morphogenesis, and defence against pathogens and abiotic stresses in bacteria, fungi, insects, plants, animals, and other organisms [30], but few studies have examined chitinases in diatoms.

Here, we investigated the chitinase gene family in the model Thalassiosira species Thalassiosira pseudonana, making use of its completely sequenced genome [16]. We identified 24 chitinase genes and analyzed their sequence structural features, scaffold locations, phylogenetic relationships, stress-related cis-acting elements, subcellular locations, and responses to abiotic stresses at the transcriptional and enzymatic levels. This is the first study to investigate the characteristics of chitinase family members in diatoms. Our results provide insight into $\beta$-chitin degradation in diatoms and can support in vitro applications of chitinases derived from diatoms.

\section{Results}

Genome-wide identification and analysis of chitinase genes in T. pseudonana

A total of 24 non-redundant chitinase genes were identified in the genome of $T$. pseudonana and designated TpChi1-24 based on their chromosomal locations. Gene names, gene IDs, chromosomal locations, exon numbers, amino acid sequence lengths, molecular weights (MW), and theoretical isoelectric points (pIs) are presented in Table 1 . The sequence lengths of the chitinases ranged from 417 (TpChi1) to 3512 (TpChi6) amino acid (aa) residues, with an average length of 1517 aa. The relative molecular mass varied from $46.4 \mathrm{kDa}$ (TpChi1) to 373.1 $\mathrm{kDa}$ (TpChi6), and the $\mathrm{pI}$ values ranged from 4.09 (TpChi6) to 5.42 (TpChi16).

Table S1 lists predicted signal peptides, transmembrane helices (TMHs), and subcellular localizations for the chitinases. HECTAR predictions indicated that up to half of them (12 out of 24) possessed signal peptides. Nine (TpChi9, TpChi13, TpChi14, TpChi17, TpChi18, TpChi20, TpChi21, TpChi22, and TpChi23) were predicted to contain signal peptides by all three programs (SignalP-5.0, HECTAR, and ASAFIND), suggesting that these chitinases are involved in the secretory pathway. Twelve (TpChi1, TpChi2, TpChi3, TpChi4, TpChi5, TpChi7, TpChi8, TpChi10, TpChi11, TpChi15, TpChi16, and TpChi19) were predicted by HECTAR to have type $\Pi$ signal anchors, indicating that they may be Type $\Pi$ transmembrane proteins with an $\mathrm{N}$-in orientation and maybe localized on chloroplast or mitochondrial membranes. Six (TpChi2, TpChi5, TpChi9, TpChi14, TpChi22, TpChi23) were predicted to contain TMHs, suggesting a subcellular localization at the plasma membrane or the endomembrane system.

\section{Gene structures, motifs, and conserved domains of the TpChis genes}

All chitinase genes contained at least two exons and one intron, and TpChi5 contained 17 exons, the most of all the chitinase genes. One quarter of the TpChis had more than ten introns $(6,25 \%)$, over one half had seven or more introns (14, 58\%), and only TpChi1 had a single intron (Fig. 1).

The sequence differences within the chitinase family were further analyzed by examining their conserved domains and motifs using the Pfam database and the MEME program, respectively. Twenty conserved motifs were identified in the T. pseudonana chitinase proteins. Their lengths varied from 15 to 65 aa, and their details are presented in Table S2. The Glyco_hydro_18 domain was identified in nineteen chitinases and the Glyco_hydro_19 domain in six. Notably, chitinase TpChi12 was found to possess both Glyco_hydro_18 and Glyco_hydro_19 domains. Three other auxiliary domains, Chitin bind_1 (PF00187), CBM_14 (Peritrophin A, PF01607), 
Table 1 Features of the identified T. pseudonana chitinase genes

\begin{tabular}{|c|c|c|c|c|c|c|c|}
\hline Name & JGI gene ID & Scaffold & Genomic location & Exon & AA & $\mathrm{MW}(\mathrm{KDa})$ & pl \\
\hline TpChil & 21,369 & 2 & chr_2:1,497,142-1,498,555 (+) & 2 & 417 & 46.40 & 4.86 \\
\hline TpChi2 & 21,501 & 2 & chr_2:2,082,322-2,089,884 (-) & 16 & 2070 & 232.39 & 5.13 \\
\hline TpChis & 21,626 & 3 & chr_3:306,112-310,877 (-) & 4 & 1464 & 159.27 & 4.64 \\
\hline TpChi4 & 21,917 & 3 & chr_3:1,759,345-1,764,662 (-) & 7 & 1498 & 165.71 & 5.21 \\
\hline TpChis & 22,088 & 4 & chr_4:199,894-204,931 (-) & 17 & 1087 & 118.17 & 4.51 \\
\hline TpChi6 & 22,238 & 4 & chr_4:960,301-971,814 (+) & 8 & 3512 & 373.14 & 4.09 \\
\hline TpChi7 & 22,376 & 4 & chr_4:1,635,653-1,638,316 (+) & 5 & 684 & 74.97 & 4.66 \\
\hline TpChis & 5406 & 5 & chr_5:1,117,857-1,121,711 (+) & 14 & 901 & 96.61 & 4.75 \\
\hline TpChig & 22,909 & 5 & chr_5:2,040,965-2,049,241 (+) & 9 & 2494 & 273.21 & 4.44 \\
\hline TpChi10 & 22,915 & 5 & chr_5:2,100,817-2,105,674 (-) & 13 & 785 & 87.47 & 4.61 \\
\hline TpChi11 & 6636 & 7 & chr_7:81,934-86,292 (+) & 5 & 1349 & 146.67 & 4.47 \\
\hline TpChi12 & 23,384 & 7 & chr_7:448,791-455,002 (-) & 8 & 1819 & 196.99 & 4.49 \\
\hline TpChi13 & 23,470 & 7 & chr_7:953,351-956,745 (+) & 3 & 1031 & 109.60 & 4.28 \\
\hline TpChi14 & 23,629 & 7 & chr_7:1,845,016-1,855,061 (-) & 15 & 2773 & 304.08 & 4.31 \\
\hline TpChi15 & 23,700 & 8 & chr_8:274,001-278,110 (-) & 7 & 1116 & 119.55 & 4.44 \\
\hline TpChi16 & 8279 & 10 & chr_10:177,498-181,104 (+) & 10 & 911 & 101.63 & 5.42 \\
\hline TpChi17 & 24,326 & $11 a$ & chr_11a:339,905-344,411 (-) & 7 & 1113 & 118.52 & 5.08 \\
\hline TpChi18 & 24,421 & 12 & chr_12:128,580-139,130 (-) & 6 & 3325 & 359.94 & 4.25 \\
\hline TpChi19 & 11,110 & 19a_19 & chr_19a_19:163,538-171,325 (-) & 8 & 2241 & 241.48 & 4.37 \\
\hline TpChi20 & 11,125 & 19a_19 & chr_19a_19:210,121-212,567 (-) & 8 & 656 & 71.64 & 4.46 \\
\hline TpChi21 & 11,236 & 19a_19 & chr_19a_19:528,078-531,770 (+) & 4 & 1122 & 121.35 & 4.59 \\
\hline TpChi22 & 11,237 & 19a_19 & chr_19a_19:532,139-535,390 (+) & 8 & 877 & 94.94 & 4.35 \\
\hline TpChi23 & 11,963 & 22 & chr_22:1,024,768-1,030,124 (-) & 13 & 1438 & 156.88 & 4.31 \\
\hline TpChi24 & 25,849 & 23 & chr_23:244567-250,756 (+) & 9 & 1735 & 187.00 & 4.66 \\
\hline
\end{tabular}

and LPMO_10 (PF03067), were detected in 10 protein sequences (41.7\%). In addition, three motif combinations corresponding to the two Glyco_hydro domains were identified. The combination of motifs 19, 11, 17, 13, and 10 was detected in the Glyco_hydro_19 domain, which was identified in chitinases TpChi1, TpChi12, TpChi13, TpChi15, TpChi19, and TpChi20. Two other highly conserved motif combinations were identified in the Glyco_ hydro_18 domain. Chitinases TpChi3, TpChi6, TpChi14, TpChi18, TpChi21, TpChi22, and TpChi24 contained the combination of motifs 18, 16, 1, 7, 3, 12 and 5, whereas TpChi2, TpChi4, TpChi5, TpChi7, TpChi8, TpChi9, TpChi10, TpChi11, TpChi12, TpChi16, and TpChi23 contained the combination of motifs $2,8,1,7$, $15,3,4,6,3$, and 9. TpChi17 was an exception, containing only motifs 1 and 14 . In general, chitinases in the same group had similar motif compositions (Fig. 1).

\section{Phylogenetic analysis of representative Thalassiosira chitinases}

An unrooted phylogenetic tree was constructed to reveal the evolutionary relationships among the chitinases of $T$. pseudonana, T. oceanica (22, Table S3) and T. weissflogii (76, Table S4). A total of 122 protein sequences from the three Thalassiosira species were used to build the phylogenetic tree (Fig. 2). The T. pseudonana chitinases were classified into eight groups (I-VII) based on the topology of the phylogenetic tree and the domain architectures of the protein sequences. GroupV was the largest and contained 6 members from T. pseudonana, 5 from $T$. oceanica, and 12 from $T$. weissflogii. A total of 15 domains were detected in the 122 chitinases from all three Thalassiosira species (Fig. 2). Groups II-VII all contained chitinases with the Glyco_hydro_18 catalytic domain, and the chitinases in GroupI (except for TwChi50) contained only the Glyco_hydro_19 domain. Some chitinases in GroupII (TwChi9, TwChi24, TwChi58, and TwChi65) contained two Glyco_hydro_18 domains. Interestingly, seven chitinases (TpChi12, TwChi11, TwChi29, TwChi31, TwChi52, TwChi59, and TwChi74) in GroupVII and TwChi50 in GroupI contained both the Glyco_hydro_18 and the Glyco_hydro_19 catalytic domains. Overall, chitinases in the same group had similar domain architectures.

\section{Scaffold locations and gene duplication of the TpChi genes}

The 24 TpChis were assigned to 12 scaffolds (Fig. 3 and Table 1), and the distribution of chitinase genes on each scaffold was uneven. Chr7 and Chr19a_19 contained the largest number of chitinase genes (4), whereas only a 


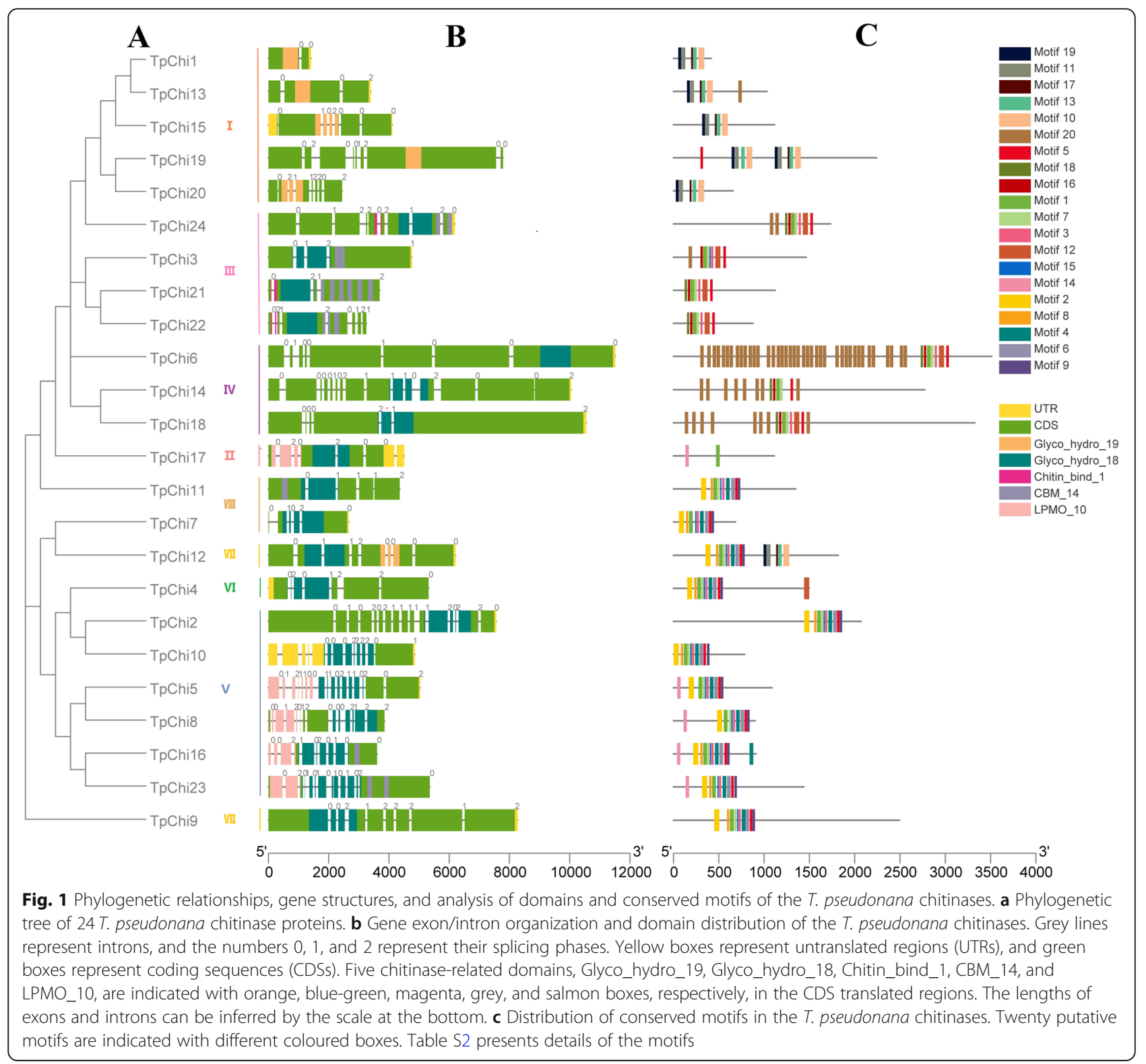

single chitinase gene was located on Chr8, Chr10, Chr11a, Chr12, Chr22, and Chr23. No segmental duplication were observed among the chitinase gene family members. However, there were two tandemly duplicated gene pairs: TpChi11 and TpChi12, and TpChi21 and TpChi22. These were located on Chr7 and Chr19a_19, respectively, and accounted for $16.7 \%$ of the chitinase genes. No collinearity among chitinase gene family members was observed with MCScanX.

\section{Stress-related cis-acting elements in the T. pseudonana chitinase gene promoters}

To investigate possible regulation mechanisms of the TpChis under abiotic or biotic stresses, $1.5 \mathrm{~kb}$ upstream sequences (promoter regions) were analyzed using the PlantCARE database to identify cis-regulatory elements. Twenty-six types of 259 stress-related cis-acting elements were identified, including light response-related (G-box and 21 other types), lowtemperature responsiveness-related (LTR), droughtinducibility-involved (MBS), and two other cis-acting elements involved in defence and stress responsiveness (i.e., the CCAAT-box and TC-rich repeats) (Fig. 4). All the chitinase genes contained 2-9 stressrelated cis-acting elements in their promoter regions. The G-box was found in all TpChi promoters and accounted for almost half of the identified elements (122). In total, 22 types of 199 light-responsive cisacting elements accounted for $76.8 \%$ of the stressrelated cis-acting elements. 


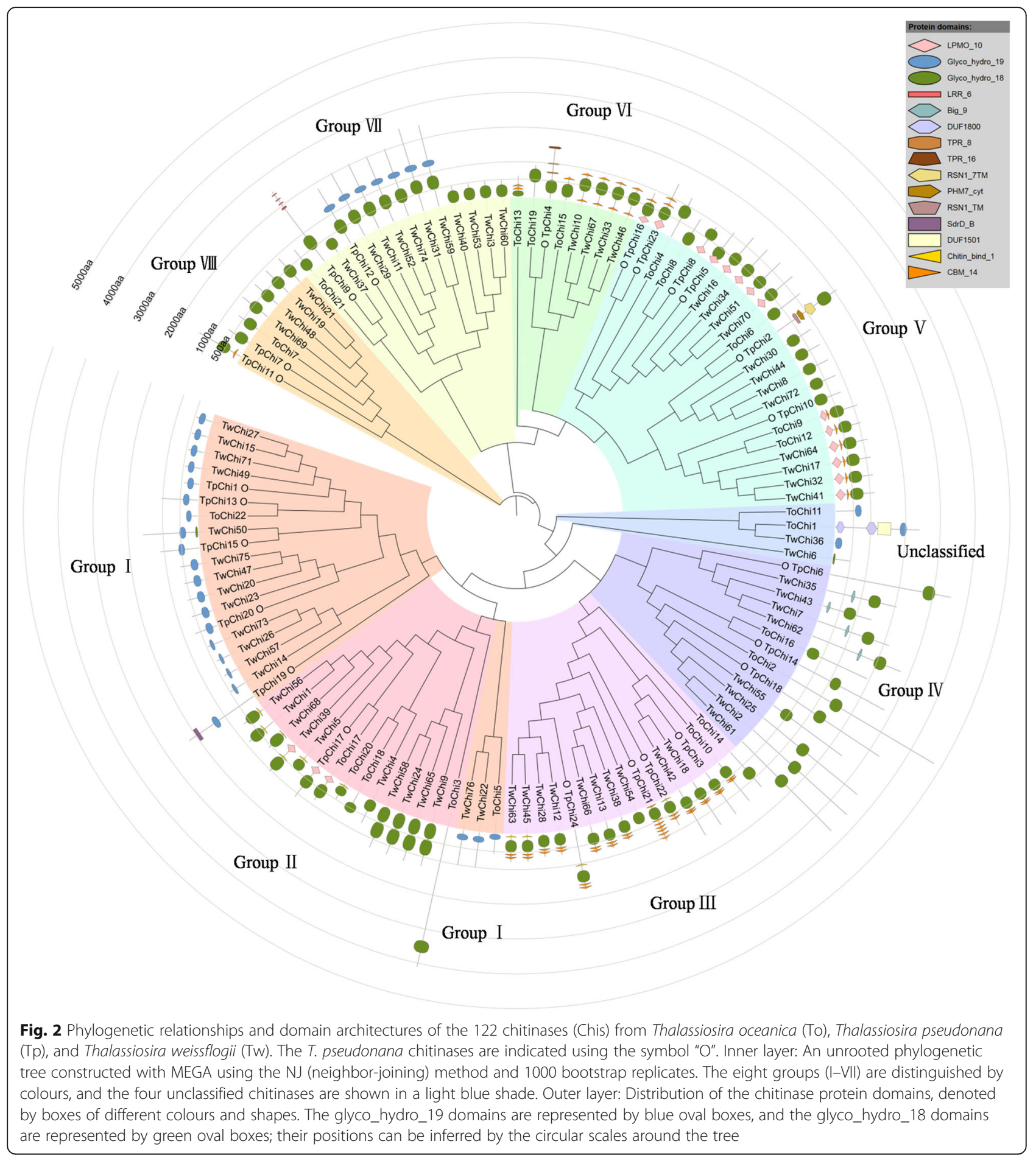

Secondary and 3D structures of the $T$. pseudonana chitinases Four secondary structures ( $\alpha$-helix, extended strand, $\beta$-turn, and random coil) were found in the $T$. pseudonana chitinases (Table S5). The $\alpha$-helix and random coil were the dominant components of chitinase secondary structure and accounted for 24.79 and $47.46 \%$ on average. Extended strand constituted $19.63 \%$ of the secondary structures and $\beta$-turn constituted $8.12 \%$. Figure 5 shows representative secondary structures for each group. 3D molecular modelling provides dynamic information that is usually difficult to acquire from experiments, and 3D protein models were constructed for the $T$. pseudonana chitinases to better understand their structural properties (Table S6). Most top-hit models (75\%) were chitin-degrading enzymes 


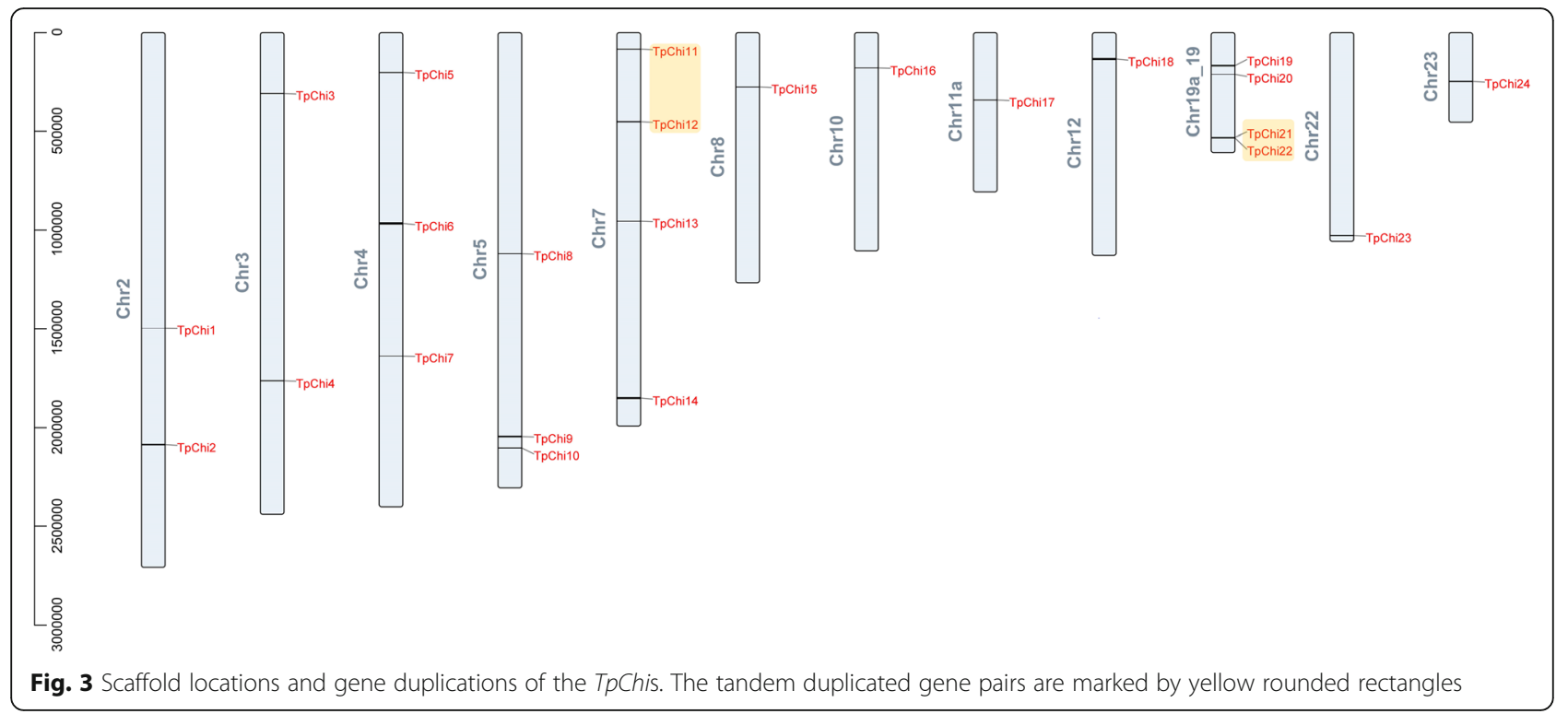

from a wide range of phylogenetic origins, including viruses (TpChi5), bacteria (TpChi2, TpChi7, TpChi8, TpChi10, TpChi11, TpChi16, TpChi17), yeast (TpChi20), fungi (TpChi12, TpChi23), rice (TpChi1, TpChi19), insects (TpChi14, TpChi18), and humans (TpChi3, TpChi21, TpChi24). T. pseudonana chitinases in the same phylogenetic groups (Fig. 2) were modelled with similar 3D structures (Table S6).

\section{Expression analysis of TpChi genes under abiotic stresses}

To investigate individual gene responses to low irradiance $\left(50 \mu \mathrm{mol} \mathrm{m}{ }^{-2} \mathrm{~s}^{-1}\right)$, low temperature $\left(12{ }^{\circ} \mathrm{C}\right)$, and silica deficiency stress at the transcriptional level, 14 genes were randomly selected for quantitative real-time polymerase chain reaction (qRT-PCR) analysis from the 24 genes that were involved in abiotic stress responses according to the cis-acting element prediction. Generally, $T p C h i$ s were upregulated after $48 \mathrm{~h}$ of exposure to the stresses (Fig. 6). Silica depletion produced the greatest increases in transcript abundance for 12 of the 14 genes $(85.7 \%)$ upregulated under silica limitation. The expression levels of $9(64.3 \%)$ and 11 (78.6\%) genes were enhanced under light-limited and temperature-limited stresses, respectively. Although most genes were

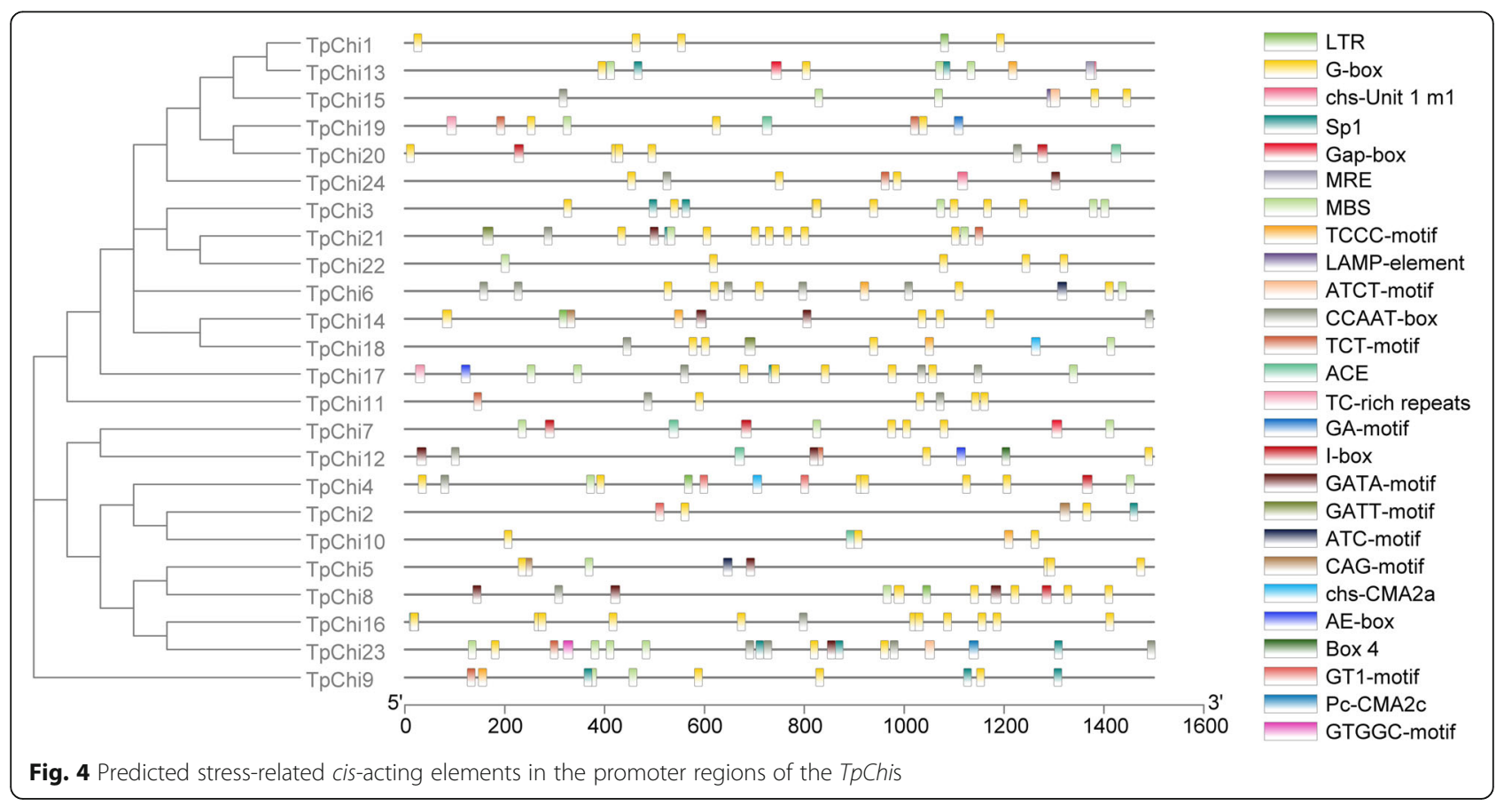




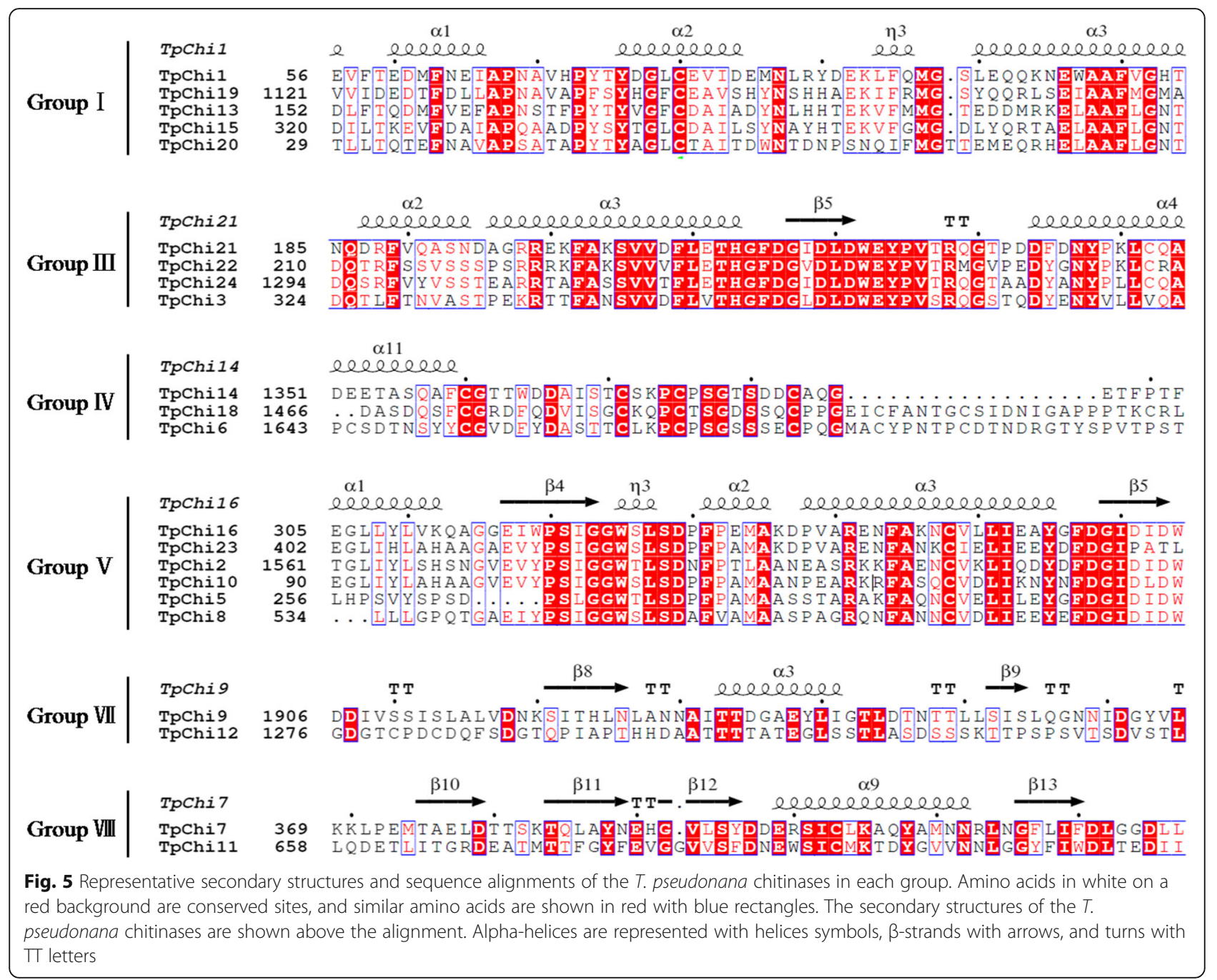

upregulated under stress, the most remarkably upregulated gene was $T p C h i 4$, whose relative expression level increased 14-, 5-, and 5-fold under light-limited, temperature-limited, and silica-limited conditions compared with the control. By contrast, only one chitinase gene (TpChi17) was down regulated overall, decreasing to one-tenth of the control expression level under temperature-limited condition.

To further investigate the involvement of the chitinase gene family in abiotic stress responses at the enzymatic level, we measured chitinase activity of $T$. pseudonana under conditions of low irradiance, low temperature, and silica depletion. The activity of the T. pseudonana chitinases remained steady under low irradiance, low temperature, and silica deficiency $(p>0.05)$ (Fig. 6).

\section{Discussion}

Chitin is the predominant polysaccharide in the diatom cell wall, and its various functions permit diatoms to adjust to the changeable ocean environment. Chitinase, the enzyme that degrades chitin into more valuable derivatives with shorter sugar chains and lower molecular weights, therefore deserves more attention in diatoms. Although the chitinase gene family has been widely investigated in insects, higher plants, and fungi [26, 27, 31, 32 ], there is little information about this gene family in Stramenopiles, and a systematic investigation has yet to be undertaken. The completeness of the $T$. pseudonana genome sequence enables the identification of putative diatom chitinase genes [16]. In the present study, T. pseudonana chitinase genes were identified from genome sequences, and their phylogenetic relationships within Thalassiosira, gene structures, conserved motifs and domains, scaffold locations, duplication events, stress-related cis-acting elements, and transcriptional expression profiles and enzymatic activities under abiotic stresses were investigated in an integrated manner. 


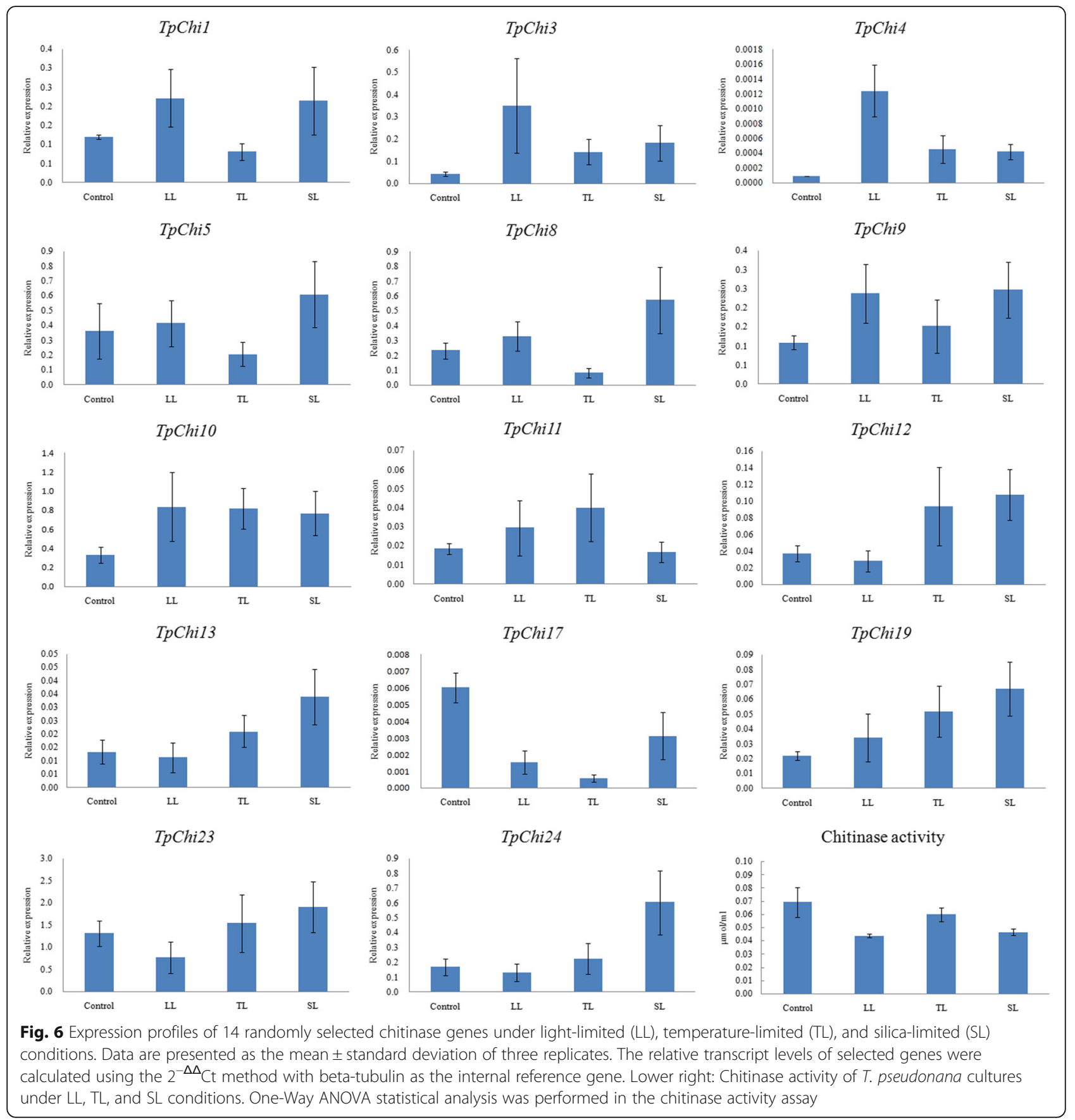

\section{Diversity in structure and intron abundance may generate chitinases with various functions in $T$. pseudonana}

In total, 24 chitinase genes were retrieved from the $T$. pseudonana genome and verified based on the identification of conserved chitinase domains. Similar numbers of chitinase genes have been reported previously in $T$. pseudonana (more than 20 chitinase-encoding genes) [33] and Cyclotella cryptica (22 chitinases) [17]. Members of the $T$. pseudonana chitinase family displayed different secondary and 3D structures (Tables S5, S6), suggesting that they may function in multiple biological processes, such as stress-related responses, growth, and developmental operations. Intron gain and loss amplifies the complexity of gene organization [34], and we found diverse exon-intron organization in the $T$. pseudonana chitinase genes (Fig. 1) that may have contributed to their structural diversity during evolution. Importantly, the TpChis generally possessed a large number of introns. Twenty-five percent had more than 10 introns, 
over half had 7 or more introns (14,58\%), and members of GroupV had the most abundant introns (Fig. 1). When abundant introns are embedded in gene sequences, alternative splicing (AS) is likely to occur during gene transcription. In eukaryotes, AS spatially and temporally regulates gene expression by selectively preserving or removing some exons, thereby producing multiple mRNAs [35]. The many possible AS variants of the TpChis may therefore enlarge the diversity of the $T$. pseudonana chitinase transcriptome and proteome.

\section{Tandem duplication and genetic diversification may co- affect the evolution of the TpChi gene family}

T. pseudonana chitinase genes were classified into eight groups (I-VII) based on a phylogenetic tree that also included proteins from two additional Thalassiosira species. Chitinases in each group displayed similar domain architectures, motif compositions (Fig. 2), and conserved secondary (Fig. 5) and 3D structure (Table S6), suggesting that they may have a closer evolutionary history and similar cellular functions.

Over the course of evolution, segmental and tandem duplications have propelled the expansion of gene families [36]. Here, we found two tandemly duplicated pairs of T. pseudonana chitinase genes (TpChil1 and TpChi12, TpChi21 and TpChi22) and no segmental duplication events, showing that tandem duplication has played a more important role in the expansion of the $T$. pseudonana chitinase gene family. TpChi21 and TpChi22 were located close together on Chr19a_19; they were classified into GroupIII (Figs. 2 and 3), and exhibited conserved domains and motif compositions (Fig. 1), as well as similar secondary and 3D structures (Fig. 5 and Table S6). The other pair, TpChi11 and TpChi12, were distantly located on Chr_7 (362 kb) and were classified into different phylogenetic groups. Chitinases TpChi11 and TpChi12 contained a common Glyco_hydro_18 domain. However, TpChi12 also possessed a Glyco_hydro_19 domain and a longer sequence length. Genetic and functional divergence of duplicated genes are known to occur during evolution [37]. TpChi12 was identified as a tandem duplicate of TpChi11, although it had an additional chitin-degrading domain. These observations suggest either the acquisition of a Glyco_hydro_ 19 domain by TpChi12 or its loss in TpChi11. Furthermore, the exon-intron structures and the gene expression profiles under silica limitation differed between TpChi11 and TpChi12, indicating that this gene pair has undergone functional divergence. Based on these results, we speculate that the chitinase gene family may have undergone duplication first, then experienced genetic diversification that drove further functional diversification.

\section{Several subcellular locations are predicted for the TpChi proteins}

Chitin fibrils are located between the silica frustule and the cell membrane of chitin-producing diatoms [38], but the locations of chitin degradation sites are unknown. We therefore investigated the subcellular locations of the $T$. pseudonana chitinases. We mainly interpreted the subcelluar locations of the TpChi proteins according to the results of HECTAR that is professionally designed for heterokonts. HECTAR predictions indicated that half of the chitinase family members are involved in the secretory pathway, consistent with the finding that a majority of chitinase genes in B. rapa and Arabidopsis thaliana are secretory $[24,27]$. This suggests that chitinases have extracellular activity and important in vitro applications.

The secretory chitinases are able to degrade the chitin fibre on the diatom cell walls, and further alter cell biosilica deposition and buoyancy [11, 12]. Moreover, in higher plants, secretory chitinases are a group of pathogenesis-related (PR) proteins that in response to pathogens and abiotic stresses [39-41]. In indica rice, transgenic rice lines and their progenies overexpressing a bitter melon chitinase gene showed enhanced resistance to major fungal pathogens of rice [40]. In winter rye, dual function-ing antifreeze proteins were observed to be homologous to apoplastic chitinases [41]. Accordingly, we can speculate that the secretory T. pseudonana chitinases were involved in the response to chitinconstituting pathogens and abiotic factors, which were also supported by the qRT-PCR results. The remaining chitinases, however, were predicted to reside on the chloroplast or mitochondrial membranes. Given the secondary endosymbiosis exhibited by diatoms [42], $T$. pseudonana chitinases anchored to the chloroplast or mitochondrial membranes may be encoded by genes originally acquired from red algae or non-photosynthetic exosymbionts.

\section{Different chitinase gene expression patterns may support chitin metabolism and other physiological needs of $T$. pseudonana in changeable environments}

Twenty-six types of stress-related cis-regulatory elements were found in the promoter regions of the 24 TpChi genes (Fig. 4), indicating that they may undergo complex transcriptional regulation. A total of 199 lightresponsive cis-acting elements were unevenly distributed in the TpChi gene promoters, suggesting that the expression of most chitinase genes was indispensably induced by light. Consistent with this observation, LTR, TC-rich repeat, and MBS elements are also important for the induction of chitinase genes in cucumber, $B$. rapa, B. juncea, and Camelina sativa [27, 28, 31]. 
Multiple studies have demonstrated variations in the transcription of chitinase family genes during biotic disease resistance in plants $[27,28,31]$. Cao \& Tan (2019) investigated the expression of tomato chitinase genes in response to the pathogen Sclerotinia sclerotiorum and under low temperature, high temperature, drought, and salt stresses [29]. They observed that the expression of chitinase genes was broadly elevated under these stress conditions. The present study demonstrated a similar pattern of overall upregulation of TpChi genes in response to low irradiance, low temperature, and silica depletion. The different transcriptional profiles of $T$. pseudonana chitinase genes under different abiotic stress conditions suggest that they have various response and regulation mechanisms and may be involved in a variety of physiological processes.

No significant change in in vivo chitinase activity was detected in response to abiotic stresses, despite the increase in chitinase gene transcripts. This can probably be ascribed to the restriction in enzymatic activity or a decrease in the translation of chitinase proteins in adverse environments. The inconsistency between gene transcription and enzyme activity may support the proposal that $T$. pseudonana chitinase genes are involved in other cellular processes apart from chitin metabolism, or it may represent a compensatory adjustment of chitin metabolism in shifting environments. The inconsistent results also suggest the possibility of post-transcriptional regulation during chitin degradation in T. pseudonana. By contrast, the expression of seabuckthorn chitinase genes was elevated at both the transcriptional [43, 44] and protein activity levels [43, 44] under cold stress. Moreover, chitinase activity of cucumber and tobacco also increased after treatment with biotic or abiotic stress inducers [45]. The results presented here suggest that there may be functional differences in the regulation and activity of chitinases in diatoms compared to those of higher plants.

Durkin et al. (2009) discovered that silica deposition and chitin exposure on the T. pseudonana cell wall were inversely correlated [33]. They suggested that chitin biosynthesis is enhanced in stressed cells that are unable to precipitate silica. In addition, co-regulation of transcript quantity by silicic acid and iron depletion is reported to be associated with cell wall processes [46]. In the present study, chitinase transcripts accumulated while enzymatic activity remained steady under silica depletion. Therefore, stable chitinase activity and higher chitin accumulation may allow chitin to serve as a substitute cell wall material when silica cannot be deposited. Moreover, as silica is required for diatom cell growth, chitin metabolism may also be affected by the abnormal growth of cells, further affecting cycles of carbon and other nutrients in the ocean [47]. However, further investigation of other genes involved in chitin metabolism (e.g., chitin synthases and chitin deacetylases) is required.

\section{Auxiliary domains may assist in chitin binding by the $T$. pseudonana chitinases}

Most chitinases contain a catalytic domain and additional auxiliary domains such as carbohydrate-binding modules (CBMs) [48], which are classified into 87 families at the CAZy database (http://www.cazy.org/). The CBMs anchor the enzyme to the substrate and disrupt its crystalline structure, creating free chain ends and thereby enhancing the enzyme's catalytic activity towards insoluble substrates [48, 49]. Chitin binding has been demonstrated for a protein containing only two CBM_14 domains [50]. In the present study, the CBM 14, Chitin_bind_1, and LMPO_10 domains, which are typically found in chitin-binding proteins, were identified in the protein sequences of chitinase TpChi3, TpChi5, TpChi8, TpChi11, TpChi16, TpChi17, TpChi21, TpChi22, TpChi23, and TpChi24 (41.7\%), five of which possessed two or more CBM_14 domains (TpChi3, TpChi21, TpChi22, TpChi23, and TpChi24) (Fig. 1), suggesting chitin-binding ability of T. pseudonana chitinases. Moreover, seven chitinases (TpChi3, TpChi11, TpChi16, TpChi21, TpChi22, TpChi23, and TpChi24) that contained the CBM_14 domain were predicted to be extracellular (containing a signal peptide) or located in the chloroplast or mitochondrial membrane (Table S1). This finding suggests more subcellular location of diatom chitinases containing the CBM_14 domain than the space between the plasma membrane and the silica frustule of diatoms as Traller et al. (2016) discussed [17].

\section{Conclusions}

We identified 24 TpChi genes from the T. pseudonana genome and divided them into eight subgroups. The gene family was analyzed from the perspective of gene structure, phylogeny, scaffold location, gene duplication, secondary and 3D structures, and expression at the transcriptional and enzymatic levels under abiotic stresses. In general, chitinase transcript levels were upregulated but enzymatic activity remained unchanged under abiotic stresses. These findings suggest that the $T$. pseudonana chitinase gene family is crucial for responses to changeable environments and plays an indispensable as yet uncharacterized role in cellular processes. Our report contributes to a better understanding of chitin metabolism in diatoms and paves the way for future functional characterization of the TpChi genes and the in vitro application of $T$. pseudonana chitinases. 


\section{Methods}

\section{T. pseudonana cultivation}

T. pseudonana (CCMP 1335) was obtained from the Microalgae Collection Center at Ningbo University (Ningbo, Zhejiang, China). Cells were grown in optimized f/2 liquid medium supplied by Shanghai Guangyu Biological Technology Co., Ltd. Cultures were grown at $19^{\circ} \mathrm{C}$ with a $12 \mathrm{~h}: 12 \mathrm{~h}$ light: dark diurnal cycle $\left(100 \mu \mathrm{mol} \mathrm{m}{ }^{-2} \mathrm{~s}^{-1}\right)$ and shaken at $100 \mathrm{rpm}$.

When cells grown in the starter culture had reached the exponential phase, $50 \mathrm{~mL}$ of cell culture were centrifuged under sterile conditions at $2850 \mathrm{~g}$ for $15 \mathrm{~min}$ (Beckman Coulter, Allegra X-I5R Centrifuge), washed once with medium, and inoculated into $50 \mathrm{~mL}$ of fresh medium. For temperature-limited treatment, cells were placed under a low temperature of $12{ }^{\circ} \mathrm{C}$ with $100 \mu \mathrm{mol}$ $\mathrm{m}^{-2} \mathrm{~s}^{-1}$ of light, whereas for the light-limited treatment, cells were exposed to a low irradiance of $50 \mu \mathrm{mol} \mathrm{m} \mathrm{m}^{-2}$ $\mathrm{s}^{-1}$ at $19^{\circ} \mathrm{C}$. For the silica-limited treatment, cells were washed once with medium and inoculated into $50 \mathrm{~mL}$ of fresh, silica-free medium and were then cultured under normal light $\left(100 \mu \mathrm{mol} \mathrm{m}{ }^{-2} \mathrm{~s}^{-1}\right)$ and temperature $\left(19{ }^{\circ} \mathrm{C}\right)$ conditions with the control group. The experiment was performed in flasks for $48 \mathrm{~h}$, and there were three biological replicates per treatment.

\section{Identification of chitinase family genes in T. pseudonana} The nucleotide sequences and corresponding protein sequences of T. pseudonana chitinase genes were retrieved from the Joint Genomics Institute PhycoCosm database (JGI PhycoCosm, https://jgi.doe.gov/data-and-tools/ phycocosm/) using "chitinase" and other relevant keywords listed in Table S7. A total of 93 hits were annotated as chitinase-relevant genes. From these, we removed 41 genes that were redundantly annotated as full-length sequences but were in fact partial sequences of the other 52 genes (Table S8). Protein sequences of the remaining 52 genes were submitted to the SMART and Pfam websites to confirm the presence of chitinase domains Glyco_hydro_18 (PF00704) and Glyco_hydro_ 19 (PF00182) with a cut-off $E$-value of $<0.0001$ [51-53]. Proteins with one or both of the Glyco_hydro_18 and Glyco_hydro_19 domains were regarded as putative chitinases.

\section{Sequence analysis and structural characterization}

All of the nucleotide sequences were analyzed using the ExPASy ProtParam tool to calculate their amino acid numbers, molecular weights, andpIs [54]. Genomic locations, intron numbers, and gene structure information were acquired from JGI PhycoCosm. MEME V5.1.1 online software was used to identify conserved motifs in the chitinase proteins with the following parameters: any number of repetitions, maximum of 20 motifs, and an optimum motif width of 6-200 amino acids [55]. Signal peptides and TMHs were predicted using SignalP-5.0 (http://www.cbs.dtu.dk/services/SignalP/) and TMHMM Server v.2.0 (http://www.cbs.dtu.dk/services/ TMHMM-2.0/), respectively. Subcellular locations were predicted by combining the results of WoLF PSORT (https://wolfpsort.hgc.jp/) with those of HECTAR and ASAFind, the two software that are specific for heterokonts [56-58].

Secondary structures of $T$. pseudonana chitinase protein sequences were predicted using SOPMA (https:// npsa-prabi.ibcp.fr/cgi-bin/npsa_automat.pl?page $=$ / NPSA/npsa_sopma.html) with default parameters. For each phylogenetic group of $T$. pseudonana chitinases that contained at least two members, protein sequences were aligned with ClustalW (https://www.genome.jp/ tools-bin/clustalw), and representative secondary structures were then visualized with ESPript3.0 [59]. 3D models of the $T$. pseudonana chitinases were constructed using the SWISS-MODEL server [60-64].

\section{Scaffold localization and gene duplication identification}

TBtools software was used to perform scaffold localization based on retrieved genomic location information [65]. Gene duplication events within the family were identified with BLASTp and MCScanX methods [66]. All the T. pseudonana chitinase protein sequences were aligned with each other using BLASTp. The BLASTp result was used as input for MCScanX, and duplication events and gene collinearity were identified using default parameters.

\section{Phylogenetic analysis and classification}

To study the evolutionary relationships among chitinases from T. pseudonana, Thalassiosira oceanica, and Thalassiosira weissflogii, an unrooted neighbor-joining phylogenetic tree was constructed using MEGA X software (v10.1.8) with 1000 bootstrap replicates [67]. The T. oceanica protein sequences were identified and downloaded from the JGI PhycoCosm database using the keyword "chitinase". To acquire T. weissflogii protein sequences, the $24 T$. pseudonana chitinase protein sequences were used as BLASTp queries against the $T$. weissflogii transcriptomes released by the Marine Microbial Eukaryote Transcriptome Sequencing Project (MMETSP) [68]. The T. oceanica and T. weissflogii candidate sequences were submitted to SMART and Pfam to verify the presence of one or both of the Glyco_hydro_18 and Glyco_hydro_19 domains [51-53]. In total, 22 full-length $T$. oceanica chitinases (Table S3), $76 T$. weissflogii chitinases (Table S4), and the 24 newly identified chitinases from T. pseudonana were used for phylogenetic analysis. 


\section{Prediction of cis-acting elements}

To predict the cis-acting regulatory elements in the promoter regions of $T$. pseudonana chitinase genes, $1.5 \mathrm{~kb}$ of sequence upstream from each initial codon (ATG) was downloaded from the JGI PhycoCosm database. Stress response-related cis-acting elements in the promoter sequences of the chitinase genes were then investigated using the PlantCARE website (http:// bioinformatics.psb.ugent.be/webtools/plantcare/html/) [69].

\section{Enzyme assays and transcriptional profile analysis}

After $48 \mathrm{~h}$ of stress treatment, a $1 \mathrm{~mL}$ sample was taken from each flask for measurement of chitinase activity using the Solarbio Chitinase Assay Kit (Cat\#BC0820) following the manufacturer's instructions. The remaining $49 \mathrm{~mL}$ of $T$. pseudonana cells in each flask were collected by centrifugation at $2850 \mathrm{~g}$, washed once with fresh medium, and flash-frozen in liquid nitrogen. The T. pseudonana cell pellets were homogenized by vortexing in TRIzol reagent (Invitrogen, Waltham, MA, USA), then centrifuged at $9700 \mathrm{~g}$. The supernatants were used for RNA extraction with chloroform and isopropanol. RNA pellets were resuspended in diethyl pyrocarbonatetreated water, followed by elimination of genomic DNA and synthesis of complementary DNA (cDNA) with a PrimeScript RT reagent kit (Takara, Japan). The synthesized cDNA was then used for qRT-PCR experiments.

The transcriptional profiles of 14 randomly selected $T$. pseudonana chitinase genes were obtained by qRT-PCR using TB Green ${ }^{\text {Tw }}$ Premix Ex Taq $^{\text {TM }}$ II (Takara, Japan) and a Thermal Cycle Dice ${ }^{\mathrm{T} x}$ Real Time System (Takara, Japan). Gene-specific qRT-PCR primers were designed using the NCBI Primer-BLAST website [70]. Information on primers is provided in Table S9. The beta tubulin gene (TUB3) was used as the reference gene for normalizing the expression of the T. pseudonana chitinase genes [71].

\section{Supplementary Information}

The online version contains supplementary material available at https://doi. org/10.1186/s12870-021-02849-2.

Additional file 1: Table S1. Analysis and prediction of signal peptides, Transmembrane helices (TMHs) and Subcellular localizations of the TpChi proteins.

Additional file 2: Table S2. List of the putative motifs of the TpChi proteins.

Additional file 3: Table S3. The information of chitinases from $T$. oceanica.

Additional file 4: Table S4. The information of chitinase from $T$. weissflogii.

Additional file 5: Table S5. The proportion of secondary structure of the TpChi proteins.
Additional file 6: Table S6. Top 3D models predicted of of the TpChi proteins.

Additional file 7: Table S7. Keywords used for searching T. pseudonana chitinase genes on JGl.

Additional file 8: Table S8. All transcripts annotated as chitinase in T. pseudonana genome from JGI database.

Additional file 9: Table S9. Primers used in QRT-PCR studies.

\section{Abbreviations}

AA: Amino acid; AS: Alternative splicing; CBMs: Carbohydrate-binding modules; JGI PhycoCosm: Joint Genomics Institute PhycoCosm database; MW: Molecular weight; NCBI: National Center for Biotechnology Information; NJ: Neighbor-joining; ORFs: Open reading frames; pl: Isoelectric point; qRTPCR: Quantitative real-time polymerase chain reaction;

TMHs: Transmembrane helices; UTR: Untranslated region; 3D: Three dimensional

\section{Acknowledgements}

The authors would like to thank TopEdit (www.topeditsci.com) for its linguistic assistance during the preparation of this manuscript. We would also like to thank Guanjie Zhang from Xi'an Jiaotong University and Beining Xue from the Institute of Oceanology, CAS, for their statistical analysis assistance and encouragements.

\section{Authors' contributions}

$\mathrm{HC}, \mathrm{ZS}$ and DD conceived and designed research. $\mathrm{HC}$ conducted experiments, analysed the data and wrote the manuscript. ZS and DD gave critical revision of the manuscript. CL analysed the data. All authors have read and approved the manuscript.

\section{Funding}

This work received financial support from the National Natural Science Foundation of China (41806175), National Key R\&D Program of China (2018YFD0900106) and Youth project of Marine Biology and Biotechnology Laboratory in Pilot National Laboratory for Marine Science and Technology Project (YQ2018NO06). These funders financially supported the instruments and reagents purchase for the research. Each of the funding bodies took part in designing the study, collecting, analysing and interpreting the data, and writing the manuscript.

\section{Availability of data and materials}

The reference genome of T. pseudonana was released in JGI PhycoCosm with the accession number of Thalassiosira pseudonana CCMP 1335 (https:// jgi.doe.gov/data-and-tools/phycocosm/). Gene sequences and protein sequences of $T$. pseudonana chitinase were downloaded from JGl PhycoCosm (https://jgi.doe.gov/data-and-tools/phycocosm/). Chitinase protein sequences of T. oceanica were downloaded from JGI PhycoCosm (https://jgi.doe.gov/data-and-tools/phycocosm/). Chitinase protein sequences of T. weissflogii were downloaded from iMicrobe website (https://www. imicrobe.us/). All of the datasets supporting the results of this article are included within the article and its additional files.

Ethics approval and consent to participate Not applicable.

\section{Consent for publication}

Not applicable.

\section{Competing interests}

The authors declare that they have no competing interests.

\section{Author details}

'Key Laboratory of Experimental Marine Biology, Center for Ocean Mega-Science, Institute of Oceanology, Chinese Academy of Sciences, Qingdao 266071, P. R. China. ${ }^{2}$ Laboratory for Marine Biology and Biotechnology, Pilot Qingdao National Laboratory for Marine Science and Technology, Qingdao 266237, P. R. China. ${ }^{3}$ University of Chinese Academy of Sciences, Beijing 100049, P. R. China. ${ }^{4}$ State Key Laboratory of Bioactive 
Seaweed Substances, Qingdao Bright Moon Seaweed Group Co Ltd, Qingdao 266400, P. R. China.

\section{Received: 23 October 2020 Accepted: 21 January 2021 Published online: 10 February 2021}

\section{References}

1. Pareek N, Vivekanand V, Singh R. Chitin deacetylase: characteristic molecular features and functional aspects. In: Shukla P, Pletschke Bl, editors. Advances in enzyme biotechnology. New Delhi: Springer; 2013. p. 125-36.

2. Shigemasa Y, Minami S. Applications of chitin and chitosan for biomaterials. Biotechnol Genet Eng Rev. 1996;13(1):383-420.

3. Yang TL. Chitin-based materials in tissue engineering: applications in soft tissue and epithelial organ. Int J Mol Sci. 2011;12(3):1936-63.

4. Rudall KM. The chitin/protein complexes of insect cuticles. Adv Insect Physiol. 1963;1:257-313.

5. Lamarque G, Cretenet M, Viton C, Domard A. New route of deacetylation of alpha- and beta-chitins by means of freeze-pump out-thaw cycles. Biomacromolecules. 2005;6(3):1380-8.

6. Cuong HN, Minh NC, Van Hoa N, Trung TS. Preparation and characterization of high purity beta-chitin from squid pens (Loligo chenisis). Int J Biol Macromol. 2016:93(Pt A):442-7.

7. Dweltz NE. The structure of $\beta$-chitin. Biochim Biophys Acta. 1961;51:283-74

8. Gaill F, Shillito B, Lechaire J, Chanzy H, Goffinet G. The chitin secreting system from deep-sea hydrothermal vent Worms. Biol Cell. 1992;76(2):201-4.

9. McLachlan J, Mcinnes A, Falk M. Studies of the chitin (chitin-poly-Nacetylglucosamine) fibres of diatom Thalassiosira fluviatilis hustedt: I. production and isolation of chitin fibres. Can J Bot. 1965:43(6):707-13.

10. Field C, Behrenfeld M, Randerson J, Falkowski P. Primary production of the biosphere: integrating terrestrial and oceanic components. Science. 1998; 281(5374):237-40.

11. Brunner E, Richthammer $P$, Ehrlich $H$, Paasch S, Simon P, Ueberlein $S$, et al. Chitin-based organic networks: an integral part of cell wall biosilica in the diatom Thalassiosira pseudonana. Angew Chem Int Ed Engl. 2009;48(51): 9724-7.

12. Walsby $A E$, Xypolyta A. The form resistance of chitan fibres attached to the cells of Thalassiosira fluviatilis Hustedt. Eur J Phycol. 1977:12(3):215-23.

13. Frischkorn KR, Stojanovski A, Paranjpye R. Vibrio parahaemolyticus type IV pili mediate interactions with diatom-derived chitin and point to an unexplored mechanism of environmental persistence. Environ Microbiol. 2013;15(5): $1416-27$.

14. Johnstone J. Conditions of life in the sea. Cambridge: University Press; 1908. p. 332.

15. Souza CP, Almeida BC, Colwell RR, Rivera IN. The importance of chitin in the marine environment. Mar Biotechnol (NY). 2011;13(5):823-30.

16. Armbrust E, Berges J, Bowler C, Green B, Martinez D, Putnam N, et al. The genome of the diatom Thalassiosira Pseudonana: ecology, evolution, and metabolism. Science. 2004;306(5693):79-86.

17. Traller JC, Cokus SJ, Lopez DA, Gaidarenko O, Smith SR, McCrow JP, et al. Genome and methylome of the oleaginous diatom Cyclotella cryptica reveal genetic flexibility toward a high lipid phenotype. Biotechnol Biofuels. 2016; 9:258.

18. Shao Z, Thomas $Y$, Hembach $L$, Xing $X$, Duan D, Moerschbacher BM, et al. Comparative characterization of putative chitin deacetylases from Phaeodactylum tricornutum and Thalassiosira pseudonana highlights the potential for distinct chitin-based metabolic processes in diatoms. New Phytol. 2019;221(4):1890-905.

19. Ortiz-Rodriguez T, de la Fuente-Salcido N, Bideshi DK, Salcedo-Hernandez R, Barboza-Corona JE. Generation of chitin-derived oligosaccharides toxic to pathogenic bacteria using ChiA74, an endochitinase native to Bacillus thuringiensis. Lett Appl Microbiol. 2010;51(2):184-90.

20. Nagpure A, Choudhary B, Gupta RK. Chitinases: in agriculture and human healthcare. Crit Rev Biotechnol. 2014;34(3):215-32.

21. Patel S, Goyal A. Chitin and chitinase: role in pathogenicity, allergenicity and health. Int J Biol Macromol. 2017;97:331-8.

22. Adrangi $S$, Faramarzi MA. From bacteria to human: a journey into the world of chitinases. Biotechnol Adv. 2013;31(8):1786-95.

23. Henrissat B. A classification of glycosyl hydrolases based on amino acid sequence similarities. Biochem J. 1991;280(2):309-16.

24. Passarinho PA, de Vries SC. Arabidopsis Chitinases: a genomic survey. The Arabidopsis Book. 2002;2002(1):e0023.
25. Hamel F, Boivin R, Tremblay C, Bellemare G. Structural and evolutionary relationships among Chitinases of flowering plants. J Mol Evol. 1997;44(6): 614-24.

26. Liu SH, Li HF, Yang Y, Yang RL, Yang WJ, Jiang HB, et al. Genome-wide identification of chitinase and chitin deacetylase gene families in the oriental fruit fly, Bactrocera dorsalis (Hendel). Comp Biochem Physiol Part D Genomics Proteomics. 2018;27:13-22.

27. Chen J, Piao Y, Liu Y, Li X, Piao Z. Genome-wide identification and expression analysis of chitinase gene family in Brassica rapa reveals its role in clubroot resistance. Plant Sci. 2018;270:257-67.

28. Bartholomew ES, Black K, Feng Z, Liu W, Shan N, Zhang X, et al. Comprehensive Analysis of the Chitinase Gene Family in Cucumber (Cucumis sativus L.): From Gene Identification and Evolution to Expression in Response to Fusarium oxysporum. Int J Mol Sci. 2019;20(21):5309.

29. Cao J, Tan X. Comprehensive analysis of the Chitinase family genes in tomato (Solanum lycopersicum). Plants. 2019;8(3):52.

30. Adrangi S, Faramarzi MA, Shahverdi AR, Sepehrizadeh Z. Purification and characterization of two extracellular endochitinases from Massilia timonae. Carbohydr Res. 2010:345(3):402-7.

31. Mir ZA, Ali S, Shivaraj SM, Bhat JA, Singh A, Yadav P, et al. Genome-wide identification and characterization of Chitinase gene family in Brassica juncea and Camelina sativa in response to Alternaria brassicae. Genomics. 2020;112(1):749-63.

32. Watanabe T, Kanai R, Kawase T, Tanabe T, Mitsutomi M, Sakuda S, Miyashita K. Family 19 chitinases of Streptomyces species: characterization and distribution. Microbiology. 1999;145:3353-63.

33. Durkin CA, Mock T, Armbrust EV. Chitin in diatoms and its association with the cell wall. Eukaryot Cell. 2009;8(7):1038-50.

34. Cao J, Li X. Identification and phylogenetic analysis of late embryogenesis abundant proteins family in tomato (Solanum lycopersicum). Planta. 2015; 241(3):757-72.

35. Xie X, Wu N. Introns in higher plant genes. Chin Sci Bull. 2002:47(17):1409-15.

36. Cannon SB, Mitra A, Baumgarten A, Young ND, May G. The roles of segmental and tandem gene duplication in the evolution of large gene families in Arabidopsis thaliana. BMC Plant Biol. 2004;4:10

37. Moore RC, Purugganan MD. The evolutionary dynamics of plant duplicate genes. Curr Opin Plant Biol. 2005;8(2):122-8.

38. Smucker R. Chitin primary production. Biochem Syst Ecol. 1991;19(5):357-69.

39. Ebrahim S, Usha K, Singh B. Pathogenesis-related (PR)-proteins: Chitinase

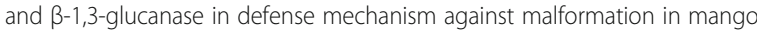
(Mangifera indica L.). Sci Hortic. 2011;130(4):847-52.

40. Li P, Pei $Y$, Sang $X$, Ling $Y$, Yang Z, He G. Transgenic indica rice expressing a bitter melon (Momordica charantia) class I chitinase gene (McCHIT1) confers enhanced resistance to Magnaporthe grisea and Rhizoctonia solani. Eur J Plant Pathol. 2009:125(4):533-43.

41. Hon W-C, Griffith M, Mlynarz A, Kwok YC, Yang DSC. Antifreeze proteins in winter Rye are similar to pathogenesis-related proteins. Plant Physiol. 1995: 109(3):879-89.

42. Prihoda J, Tanaka A, de Paula WB, Allen JF, Tirichine L, Bowler C. Chloroplastmitochondria cross-talk in diatoms. J Exp Bot. 2012;63(4):1543-57.

43. Kashyap P, Deswal R. A novel class I Chitinase from Hippophae rhamnoides: indications for participating in ICE-CBF cold stress signaling pathway. Plant Sci. 2017;259:62-70.

44. Gupta R, Deswal R. Low temperature stress modulated secretome analysis and purification of antifreeze protein from Hippophae rhamnoides, a Himalayan wonder plant. J Proteome Res. 2012;11(5):2684-96.

45. Schneider S, Ullrich WR. Differential induction of resistance and enhanced enzyme activities in cucumber and tobacco caused by treatment with various abiotic and biotic inducers. Physiol Mol Plant Pathol. 1994:45:291-304

46. Mock T, Samanta MP, Iverson V, Berthiaume C, Robison M, Holtermann K, et al. Whole-genome expression profiling of the marine diatom Thalassiosira pseudonana identifies genes involved in silicon bioprocesses. Proc Natl Acad Sci U S A. 2008:105(5):1579-84.

47. Dugdale $\mathrm{R}$, Wilkerson $\mathrm{F}$, Minas $\mathrm{H}$. The role of a silicate pump in driving new production. Deep-Sea Res I Oceanogr Res Pap. 1995:42(5):697-719.

48. Guillen D, Sanchez S, Rodriguez-Sanoja R. Carbohydrate-binding domains: multiplicity of biological roles. Appl Microbiol Biotechnol. 2010:85(5):1241-9.

49. Vaaje-Kolstad G, Horn SJ, van Aalten DM, Synstad B, Eijsink VG. The noncatalytic chitin-binding protein CBP21 from Serratia marcescens is essential for chitin degradation. J Biol Chem. 2005;280(31):28492-7. 
50. Shen Z, Jacobs-Lorena M. A type I peritrophic matrix protein from the malaria vector Anopheles gambiae binds to chitin - cloning, expression, and characterization. J Biol Chem. 1998;273(28):17665-70.

51. Letunic I, Doerks T, Bork P. SMART: recent updates, new developments and status in 2015. Nucleic Acids Res. 2015;43(Database issue):D257-60.

52. Letunic I, Bork P. 20 years of the SMART protein domain annotation resource. Nucleic Acids Res. 2018;46(D1):D493-D6.

53. El-Gebali S, Mistry J, Bateman A, Eddy SR, Luciani A, Potter SC, et al. The Pfam protein families database in 2019. Nucleic Acids Res. 2019;47(D1): D427-D32.

54. Gasteiger E, Hoogland C, Gattiker A, Duvaud S, Wilkins MR, Appel RD, et al. Protein identification and analysis tools on the ExPASy server. In: Walker JM, editor. The proteomics protocols handbook. Totowa: Humana Press; 2005. p. 571-607.

55. Bailey TL, Boden M, Buske FA, Frith M, Grant CE, Clementi L, et al. MEME SUITE: tools for motif discovery and searching. Nucleic Acids Res. 2009; 37(Web Server issue):W202-8.

56. Gschloessl B, Guermeur Y, Cock JM. HECTAR: a method to predict subcellular targeting in heterokonts. BMC Bioinf. 2008;9:393.

57. Gruber A, Rocap G, Kroth PG, Armbrust EV, Mock T. Plastid proteome prediction for diatoms and other algae with secondary plastids of the red lineage. Plant J. 2015;81(3):519-28.

58. Bendtsen JD, Nielsen H, von Heijne G, Brunak S. Improved prediction of signal peptides: SignalP 3.0. J Mol Biol. 2004;340(4):783-95.

59. Robert $X$, Gouet P. Deciphering key features in protein structures with the new ENDscript server. Nucleic Acids Res. 2014:42(Web Server issue):W320-4.

60. Guex N, Peitsch MC, Schwede T. Automated comparative protein structure modeling with SWISS-MODEL and Swiss-PdbViewer: a historical perspective. Electrophoresis. 2009;30(Suppl 1):S162-73.

61. Bertoni M, Kiefer F, Biasini M, Bordoli L, Schwede T. Modeling protein quaternary structure of homo- and hetero-oligomers beyond binary interactions by homology. Sci Rep. 2017;7(1):10480.

62. Bienert S, Waterhouse A, de Beer TA, Tauriello G, Studer G, Bordoli L, et al. The SWISS-MODEL repository-new features and functionality. Nucleic Acids Res. 2017:45(D1):D313-D9.

63. Waterhouse A, Bertoni M, Bienert S, Studer G, Tauriello G, Gumienny R, et al. SWISS-MODEL: homology modelling of protein structures and complexes. Nucleic Acids Res. 2018;46(W1):W296-303.

64. Studer G, Rempfer C, Waterhouse AM, Gumienny R, Haas J, Schwede T. QMEANDisCo-distance constraints applied on model quality estimation. Bioinformatics. 2020;36(6):1765-71.

65. Chen C, Chen H, Zhang Y, Thomas HR, Frank MH, He Y, et al. TBtools: an integrative toolkit developed for interactive analyses of big biological data Mol Plant. 2020;13(8):1194-202.

66. Wang Y, Tang H, Debarry JD, Tan X, Li J, Wang X, et al. MCScanX: a toolkit for detection and evolutionary analysis of gene synteny and collinearity. Nucleic Acids Res. 2012;40(7):e49.

67. Kumar S, Stecher G, Li M, Knyaz C, Tamura K. MEGA X: Molecular evolutionary genetics analysis across computing platforms. Mol Biol Evol. 2018:35(6):1547-9.

68. Keeling PJ, Burki F, Wilcox HM, Allam B, Allen EE, Amaral-Zettler LA, et al. The marine microbial eukaryote Transcriptome sequencing project (MMET SP): illuminating the functional diversity of eukaryotic life in the oceans through transcriptome sequencing. PLoS Biol. 2014;12(6):e1001889.

69. Lescot M, Dehais P, Thijs G, Marchal K, Moreau Y, Van de Peer Y, et al, PlantCARE, a database of plant cis-acting regulatory elements and a portal to tools for in silico analysis of promoter sequences. Nucleic Acids Res. 2002;30(1):325-7.

70. Ye J, Coulouris G, Zaretskaya I, Cutcutache I, Rozen S, Madden TL. PrimerBLAST: a tool to design target-specific primers for polymerase chain reaction. BMC Bioinf. 2012:13:134

71. Kettles N, Kopriva S, Malin G. Insights into the regulation of DMSP synthesis in the diatom Thalassiosira pseudonana through APR activity, proteomics and gene expression analyses on cells acclimating to changes in salinity, Light and Nitrogen. PLOS ONE. 2014;9(4):e94795.

\section{Publisher's Note}

Springer Nature remains neutral with regard to jurisdictional claims in published maps and institutional affiliations.

\section{Ready to submit your research? Choose BMC and benefit from:}

- fast, convenient online submission

- thorough peer review by experienced researchers in your field

- rapid publication on acceptance

- support for research data, including large and complex data types

- gold Open Access which fosters wider collaboration and increased citations

- maximum visibility for your research: over $100 \mathrm{M}$ website views per year

At BMC, research is always in progress.

Learn more biomedcentral.com/submissions 